\title{
ANÁliSE COMPARATIVA ENTRE O ÓLEO DE SOJA E O ÓLEO DE MACAÚBA NA PRODUÇÃO DE ÉSTERES ETÍLICOS EM MODO CONTÍNUO
}

\author{
C. M. T. SANTIN ${ }^{1}$, D. de OLIVEIRA $^{1}$ e J. V. de OLIVEIRA $^{1}$ \\ ${ }^{1}$ Universidade Federal de Santa Catarina, Departamento de Engenharia Química e Engenharia de \\ Alimentos \\ E-mail para contato: claudiatrentin06@yahoo.com.br
}

\begin{abstract}
RESUMO - O emprego de ultrassom apresenta-se como uma alternativa aos processos de produção de ésteres etílicos. As cavitações geradas pelo sistema aumentam a miscibilidade entre os reagentes e reduzem o tempo de reação. $\mathrm{O}$ objetivo deste trabalho foi o estudo comparativo da produção de ésteres etílicos através do processo de transesterificação do óleo de macaúba (Acrocomia aculeata) e do óleo de soja marca (Soya), utilizando o modo continuo catalisador enzimático e empregando sistema de ultrassom em meio livre de solvente orgânico. O sistema experimental utilizado consistiu num reator de polietileno de alta densidade com $1 / 16$ in de diâmetro externo, comprimento de $2 \mathrm{~m}$ e volume de $27,05 \mathrm{~mL}$, empacotado com o catalizador enzimático Novozym 435 e submerso em banho de ultrassom. Foi estudado o efeito da potência do ultrassom para a faixa de 0 e $100 \mathrm{~W}, 65^{\circ} \mathrm{C}$, razão molar (óleo/etanol) 1:6 e vazão de alimentação de substrato de $0,5 \mathrm{~mL} / \mathrm{min}$. Ao final o óleo de macaúba apresenta um melhor desempenho do que o óleo de soja.
\end{abstract}

\section{INTRODUÇÃO}

A síntese de biodiesel tornou-se um assunto atualmente de grande interesse comercial, devido à sua compatibilidade com o diesel do petróleo e seu menor impacto ambiental. As principais vantagens da utilização de biodiesel é que ele é biodegradável, pode ser utilizado sem modificar os motores atuais, e produz emissões de gases menos prejudiciais. Quimicamente, o biodiesel é uma mistura de ésteres de alquilo de ácidos gordos de cadeia longa e é normalmente produzido a partir de recursos biológicos, tais como óleos vegetais, gorduras animais (THANH et al., 2010) ou ainda os óleos de cozinha usados (ISSARIYAKUL et al., 2008) . Neste sentido, devido à baixa miscibilidade mútua dos substratos, a aplicação de irradiação ultrassônica pode ser um meio viável para ultrapassar as limitações de transferência de massa, e melhorar as taxas de reação.

A literatura apresenta vários trabalhos sobre a produção de biodiesel por meio de irradiação de ultrassom (THANH et al., 2010), mas poucos relatos recentes, foram encontrados sobre a transesterificação catalisada por enzima através da técnica de ultrassom (YU et al., 2010). De fato, tal como mencionado por Yu et al. (2010) pouca pesquisa tem sido feito até à data sobre a produção de biodiesel catalisada por lipase através de irradiação ultrassom e, portanto, a demonstração da utilidade 
técnica continua a ser uma preocupação premente. Além disso, como a síntese enzimática de biodiesel é muito mais lenta do que a transesterificação catalisada alcalina, a irradiação de ultrassom parece ser um método adequado para aumentar a velocidade de reação, tornando possível a produção de biodiesel mais verde através de um catalisador biológico.

O processo enzimático para a produção de biodiesel pode resultar em rendimento elevado de ésteres e provou ser mais tolerante à presença de um elevado teor de água e de ácidos gordos livres, o que permite o uso de vários tipos de óleos vegetais, mesmo os óleos usados (YU et al., 2010; BATISTELLA et al., 2012) e gorduras de origem animal (MARULANDA et al., 2010). O uso de matérias-primas mais baratas, tais como óleos usados, óleos vegetais crus e álcool retificado para a produção de biodiesel por meio deste processo alternativo é muito importante para a competitividade económica do biocombustível e do processo.

No Brasil cerca de $80 \%$ do biodiesel é produzido a partir de óleo de soja (MME / EPE, 2012). O preço atual do óleo de soja é cerca de US\$ 1100 por tonelada, enquanto o preço do óleo de macaúba varia de US\$ 600 a US\$ 800 por tonelada (ALICEWEB, 2013). Este cenário pode favorecer a utilização de processos enzimáticos e de matérias-primas renováveis inteiramente para a obtenção de um biocombustível acessível.

Com base nos aspectos acima indicado, este trabalho tem como objetivo investigar o potencial do óleo de macaúba como fonte para a produção de ésteres etílicos por meio da transesterificação empregando catalise enzimática em sistema livre de solvente, assistido por ultrassom e comparar os resultados obtidos com os resultados utilizando óleo de soja refinado como substrato.

\section{MATERIAIS E MÉTODOS}

\subsection{Materiais}

Nas reações de transesterificação utilizou-se como substratos o óleo de soja comercial (Marca Soya) e o óleo do fruto da macaúba (Acrocomia aculeata (Jacq.) Lodd. ex Mart) obtido da unidade de beneficiamento coco de macaúba (Associação Comunitária dos Pequenos Produtores Rurais de Riacho D'Antas e Adjacências - COOPER RIACHÃO - Montes Claros-MG), ambos os óleo sem nenhum tratamento prévio, álcool etílico (Merck, 99,9\% de pureza) e a enzima comercial Novozym 435, com atividade inicial de 47,9 U/g, produzida a partir da lipase de Candida antarctica, imobilizada em resina acrílica macro porosa de troca iônica, pela Novozymes Brasil/ Araucária-PR.

Para a determinação da atividade enzimática os seguintes reagentes/ solventes foram utlizados: acetona P.A ACS (Vetec, 99,5\%), álcool etílico (Merck, 99,9 \% de pureza) e hexano (Nuclear, P.A). Ácido láurico (Vetec, 98\% CG) e álcool n-propílico normal (Nuclear, P.A 99,5\%) foram utilizados como substratos para dosagem da atividade de esterificação das lipases. 


\section{9 a 22 de outubro de 2014 \\ Florianópolis/SC}

\subsection{Aparato e procedimento experimental}

Os experimentos para produção enzimática de ésteres etílicos foram realizados num reator de polietileno de alta densidade com 1/16 in de diâmetro externo, espessura de 1,1 mm, diâmetro interno de 4,15mm, comprimento de 2 metros e volume de 27,05mL com alimentação da mistura reacional de óleo de soja/ óleo de macaúba e álcool etílico (substrato), com uso de aproximadamente $11 \mathrm{~g}$ de catalisador, sendo este a enzima Novozym 435, um banho de ultrassom (Unique ultraSonic cleaner, modelo: USC-180A, frequência US: 40KHz, potência US: 154W).

O procedimento experimental consistiu no empacotamento do reator com a enzima Novozym 435 sob a ação de um agitador magnético, a fim de realizar um empacotamento mais eficiente, conforme Dalla Rosa et al. (2009). Após acoplou-se o reator ao sistema reacional e imergiu-o no banho de ultrassom já a condicionado na temperatura e potência ser realizado o experimento, iniciouse o bombeamento contínuo dos substratos óleo de soja/ óleo de macaúba e álcool etílico, previamente homogeneizados sob a mesma agitação durante a reação por um agitador mecânico (Marca Fisatom, Modelo 712) inserido no frasco contendo a mistura reacional, a uma dada razão molar (óleo:álcool) e vazão volumétrica de alimentação do substrato, até o completo preenchimento do sistema reacional, usando uma bomba de alta pressão para líquidos (HPLC) Digital Série III (Marca Acuflow). A bomba é utilizada para deslocar a mistura reacional para a zona de reação e manter a pressão do sistema.

As condições experimentais utilizadas neste trabalho, foram razão molar (óleo/etanol) 1:6, vazão de alimentação de substrato $0,5 \mathrm{~mL} / \mathrm{min}$, temperatura $65^{\circ} \mathrm{C}$ e potência ultrassônica de 0 e 154 Watts, estas condições foram estabelecidas com base em trabalhos anteriores realizados pelo grupo.

Quantificação dos ésteres etílicos: Para a quantificação de ésteres de ácidos graxos as amostras foram previamente preparadas, transferindo-se $250 \mathrm{mg}$ das mesmas para um balão volumétrico de 10 $\mathrm{mL}$ completando o volume até o menisco do mesmo com n-heptano. Após, transferia-se uma alíquota de $50 \mu \mathrm{L}$ desta solução para um balão volumétrico de $1 \mathrm{~mL}$ e adicionava-se $50 \mu \mathrm{L}$ do padrão interno heptadecanoato de metila (C17:0) (Sigma- Aldrich, 99,9\%) na concentração de $5000 \mathrm{mg} / \mathrm{L}$ e completava-se o volume com n-heptano. As amostras de cada experimento foram preparadas em triplicata.

A solução foi então injetada $(1 \mu \mathrm{L})$ em um cromatógrafo gasoso (GC) (Shimadzu 2010), com injetor automático (Split) e detector de ionização de chama (FID). Utilizou-se a coluna capilar RtxWAX (30 m x 0,25 mm x 0,25 mm) nas condições cromatográficas descritas pela norma EN 14103 (2003), do Comitê Europeu para Padronizações. A temperatura inicial da coluna foi $120{ }^{\circ} \mathrm{C}$ permanecendo por 1 minuto, seguido pelo aquecimento de $15^{\circ} \mathrm{C} / \mathrm{min}$ até $180{ }^{\circ} \mathrm{C}$ permanecendo por 2 minutos, e novamente aquecendo $5^{\circ} \mathrm{C} / \mathrm{min}$ até $250{ }^{\circ} \mathrm{C}$ permanecendo assim por mais 2 minutos. Nitrogênio foi utilizado como gás de arraste e a temperatura do injetor e detector foram de $250{ }^{\circ} \mathrm{C}$ e a taxa de split de 1:50. Possibilitando a determinação da conversão de triglicerídeios para ésteres etílicos da reação. 


\section{RESULTADOS E DISCUSSÃO}

Os ácidos graxos são constituintes dos óleos e gorduras na forma de mono, di e triglicerídios, uma grande quantidade de ácidos graxos livres indica que o produto está em acelerado grau de deterioração. A principal consequência disso é que o produto torna-se mais ácido. Um elevado índice de acidez indica, portanto, que o óleo ou gordura está sofrendo quebras em sua cadeia, liberando seus constituintes principais: os ácidos graxos.

De acordo com Gonzalez (2012), o óleo de macaúba apresenta as seguintes características físico-química detalhadas na Tabela 1 abaixo.

Tabela 1- Características físico-química do óleo de macaúba

\begin{tabular}{lc}
\hline \multicolumn{1}{c}{ Características físico-química } & Valores \pm desvio \\
\hline Teor de água $(\%)$ & $0,94 \pm 0,16$ \\
Índice de acidez (mg KOH.g-1) & $114,42 \pm 0,4$ \\
Índice de peróxido (meq de peróxido kg-1) & $4,4 \pm 4.10-2$ \\
Densidade (g.cm-3 a $\left.25^{\circ} \mathrm{C}\right)$ & $0,9273 \pm 6,84.10-4$ \\
Índice de saponificação $(*)$ & $204,20 \pm 2,9$ \\
\hline
\end{tabular}

* mg KOH requerido para saponificar $1 \mathrm{~g}$ de óleo

Fonte: Gonzalez, 2012.

Gonzalez (2012), realizou a determinação qualitativa das classes lipídicas por cromatografia de camada delgada do óleo de macauba, conforme Figura 28 e observou que na primeira coluna da Figura apresentam-se os padrões para monoglicerídeo (MAG), diglicerídeo (DAG), triglicerídeo (TAG), ácidos graxos livres (AGL), ésteres de ácidos graxos (biodiesel) e ceras. A segunda coluna apresentam-se os resultados da presença ou ausência dessas classes lipídicas no óleo de macaúba. Em conformidade com Gonzalez, 2012 para esse óleo verifica-se a presença de monoglicerídeo, diglicerídeo, triglicerídeo e ácidos graxos livres e a ausência de éster de ácidos graxos e ceras. Para o óleo de macaúba observa-se uma maior intensidade na mancha de ácidos graxos livres, que sugere uma maior quantidade desses compostos, coerente com o valor elevado de acidez encontrado no óleo. 


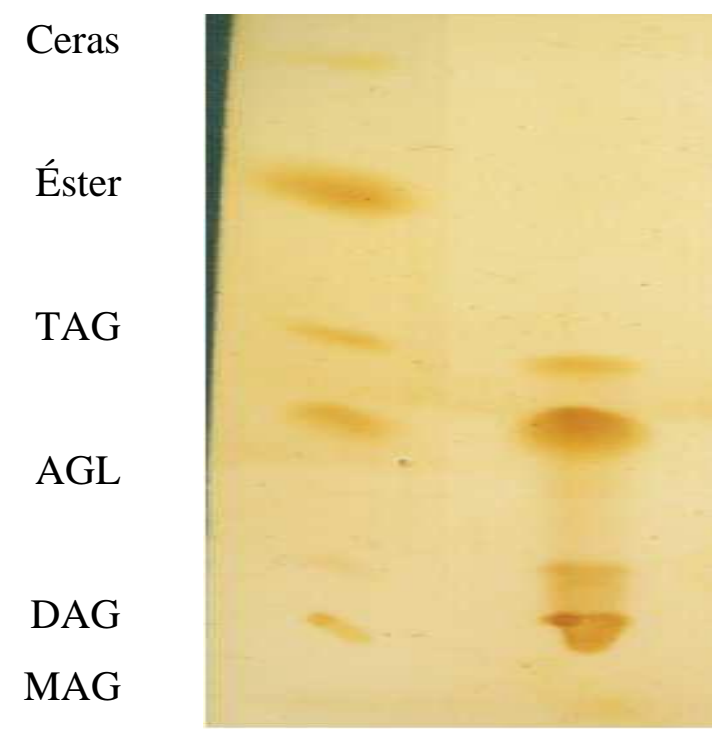

(a)

(b)

Figura 1- Identificação das classes lipídicas do óleo de macaúba por cromatografia de camada delgada: (a) padrão e (b) óleo de macaúba.

Fonte: Gonzalez (2012).

Fortes e Baugh (2004) determinaram a composição de ácidos graxos presentes no óleo da polpa de macaúba e verificaram que o ácido oléico (18:1) está presente em maior concentração - 52,82 \%, seguido do ácido palmítico (16:0) com 22,3\%, esteárico (18:0) com 5,75\%, palmitoléico (16:1) com $5,28 \%$ e linoléico (18:2) com 4,69\%, os outros ácidos graxos determinados pelos autores possuíam concentrações menores que $3 \%$.

O óleo pode sofrer várias alterações onde ocorre a formação de polímeros, dímeros, triglicerois oxidado, etc. (RUIZ-MÉNDEZ et al., 2008). Conforme Gonzalez (2012), esses compostos formados não podem ser convertidos em ésteres, implicando em um decréscimo real do rendimento da reação. A partir do método de conversão quantitativa com BF3, Gonzalez, 2012 verificou o valor máximo de conteúdo de ésteres no óleo, ou seja, o valor de convertibilidade máxima para o óleo, que no caso do óleo de macaúba foi de 75,04\%.

$\mathrm{Na}$ transesterificação do óleo de macaúba em etanol avaliou-se o efeito da potência do ultrassom, e comparou-se com a transesterificação do óleo de soja em etanol. Para a potência a faixa de estudo foi de 0 e 100 Watts, $65^{\circ} \mathrm{C}$, razão molar (óleo/etanol) 1:6 e vazão de alimentação de substrato de $0,5 \mathrm{~mL} / \mathrm{min}$. 


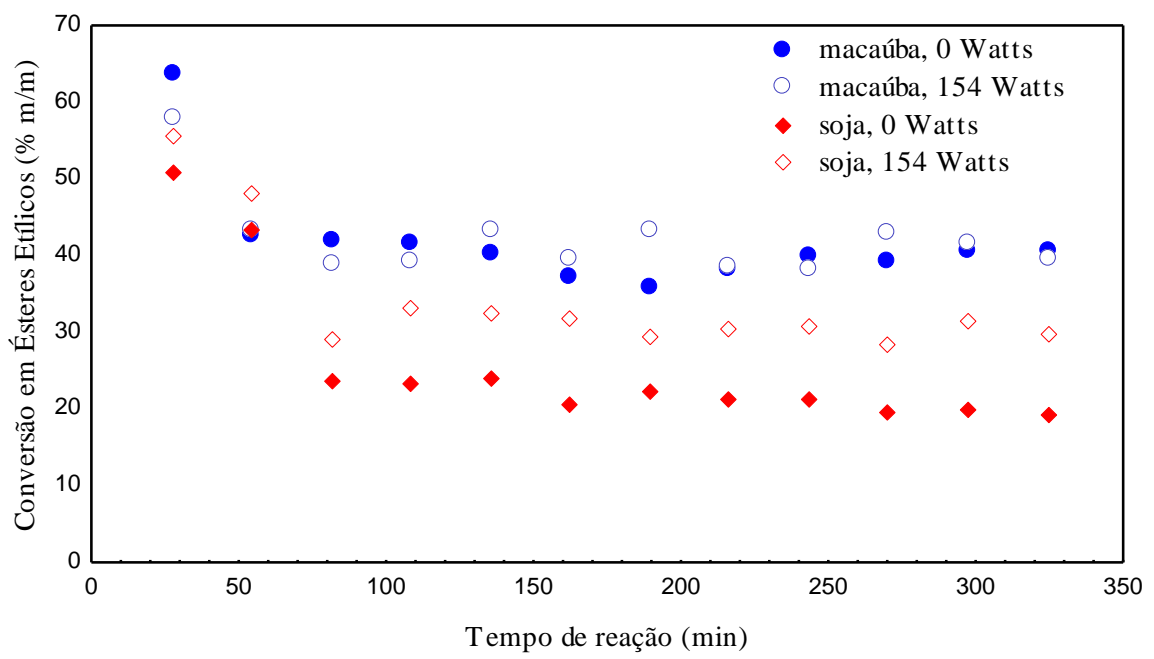

Figura 2 - Conversão de triglicerídeos do óleo de macaúba e do óleo de soja em ésteres etílicos. Razão molar (óleo/etanol) 1:6, vazão de alimentação de substrato $0,5 \mathrm{~mL} / \mathrm{min}, 65^{\circ} \mathrm{C}$, potência ultrassônica de 0 e 154 Watts.

Conforme dados apresentados pela Figura 2, verifica-se que a potência ultrassônica não exerce influência sobre conversão em ésteres etílicos quando utilizou-se o óleo de macaúba, pois levando em consideração a convertibilidade máxima do óleo de macaúba utilizado de 75,04\%, verifica-se para os tempos de reação de 27, 54, 81 e 108 minutos, potência de 0 Watts, para o óleo de macaúba conversões de 63,$73 ; 42,87 ; 42,24$ e $41,85 \%$ em ésteres etílicos sendo que está conversão se mantem em torno de $40 \%$ até o final da reação, estes resultados representam uma conversão de cerca de $85 \%$ em 27minutos, isto é, se o óleo convertesse 100\%. Para 154 Watts de potência, verifica-se conversões na ordem de 58,11;43,46;39;39,3\% em ésteres etílicos. Já para o óleo de soja pode-se observar claramente que a potência ultrassônica exerce influência na conversão, visto que para 0 Watts obtêmse uma conversão de 51,06; 43,46 e 23,84\% em ésteres para os tempos de reação de 27,54 e 81 minutos, sendo que a conversão se mantêm até o final da reação na ordem de conversão de $20 \%$ em ésteres, para 154 Watts de potência ultrassônica obtêm-se 55,60;48,39 e 29,18\% em ésteres e a conversão também se mantêm ao longo da reação em torno de $30 \%$ de conversão de triglicerídeos em ésteres. Desta forma ao realizar uma comparação entre os dois óleos (soja e macaúba), constata-se que o óleo de macaúba apresenta um melhor desempenho apesar de seu alto teor de acidez.

A Tabela 2 abaixo apresenta os valores da atividade enzimática obtida ao final de cada reação de transesterificação do óleo de soja e macaúba, para a razão molar (óleo/etanol) 1:6 e $65^{\circ} \mathrm{C}$. 
Tabela 2 - Atividade de esterificação enzimática ao final da reação de transesterificação

\begin{tabular}{ccc}
\hline Potência (Watts) & Razão molar (1:6) & Atividade Enzimática (U/g) \\
\hline 0 & Óleo de soja/ etanol & 53,65 \\
154 & Óleo de soja/ etanol & 44,33 \\
0 & Óleo de macaúba/ etanol & 46,31 \\
154 & Óleo de macaúba/ etanol & 41,17 \\
\hline
\end{tabular}

Conforme Tabela 2, a comparação dos resultados entre a atividade do biocatalisador na ausência e na presença de potencia ultrassônica mostrou que a atividade enzimática é aumentada na ausência de irradiação ultrassônica.

Nogueira et al. (2010), investigou o efeito da irradiação por microondas na taxa de transesterificação do óleo de macaúba com etanol catalisada pelas enzimas, Novozyme 435 (Candida antarctica) e Lipozyme IM (Mucor miehei). As variáveis experimentais foram temperatura (30 a $40^{\circ} \mathrm{C}$ ), tempo de reação (5 a $15 \mathrm{~min}$ ) e concentração de enzima $(2,5$ a 7,5\% m/m). Para isso misturou de $10 \mathrm{~g}$ de óleo de macaúba com $5 \mathrm{~g}$ de etanol (1:9 razão molar) numa placa de agitação durante 10 min. Os reagentes misturados com o catalisador foram então transferidos para vasos de reator de microondas (Synthos-Anton Paar) para iniciar a reação, esta foi realizada nas condições com a máxima velocidade de agitação disponível no reator. No final da reação, a mistura foi filtrada para remoção do catalisador. Nogueira et al. (2010), observou conversões de 45,2\% para a enzima Novozym 435, para as condições de $30^{\circ} \mathrm{C}, 2,5 \% \mathrm{~m} / \mathrm{m}$ concentração de enzima e 15 min de reação e de $35,8 \%$ para a Lipozyme IM a $40{ }^{\circ} \mathrm{C}, 7,5 \% \mathrm{~m} / \mathrm{m}$ de enzima e 5 minutos de reação, o tempo de reação apresentou um efeito significativo na redução da atividade catalítica da enzima, que foi interpretada em termos de desativação da enzima devido a exposição a microondas.

\section{CONCLUSÃO}

Ao final deste trabalho conclui-se que o óleo de macaúba apresentou melhor conversão em ésteres etílicos $63,7 \%$ em um tempo reacional relativamente curto de 27 minutos e o uso do ultrassom no processo de produção enzimática de ésteres etílicos em sistema livre de solvente não contribuiu para o aumento das conversões em ésteres etílicos quando utilizado o óleo de macaúba visto que para o tempo reacional de 54 min e potências de $0 \mathrm{~W}$ e $154 \mathrm{~W}$ obteve-se 42,8\% e 43,4\% em ésteres, porém para o óleo de soja a irradiação ultrassônica apresentou efeitos sendo que se observou um aumento nas conversões de $43 \%$ para $48 \%$ para as mesmas condições destacadas a cima, porém a irradiação ultrassônica afeta o biocatalizador reduzindo sua atividade enzimática.

As diferenças de comportamento de ambos os óleos (soja e macaúba) é devido às características físico-químicas principalmente a devido à acidez dos óleos. Diante dos dados obtidos verifica-se que o óleo de macaúba apresenta maior potencial para a produção de biodiesel além de ser um óleo não comestível não afetando assim a matriz energética alimentícia. 


\section{REFERÊNCIAS}

Análise das Informações do Comércio Exterior (ALICEweb), Ministério do Desenvolvimento, Indústria e Comércio Exterior (MDIC-Brasil), (2013) Import/Export 1997-2013, Retrieved April 24, 2013 from <http://aliceweb2.mdic.gov.br/index/home\#>.

BATISTELLA L.; LERIN L.A.; BRUGNEROTTO P.; DANIELLI A.S.; TRENTIN C.M.; POPIOLSKI A.; TREICHEL H.; OLIVEIRA J.V.; OLIVEIRA D. Ultrasound-assisted lipasecatalyzed transesterification of soybean oil in organic solvent system. Ultrason Sonochem., v. 19, p. 452, 2012.

DALLA ROSA, C.; MORANDIM, M.B.; NINOW, J.L.; OLIVEIRA, D.; TREICHEL, H.; OLIVEIRA, J.V. Continuous lipase-catalyzed production of fatty acid ethyl esters from soybean oil in compressed fluids. Bioresource Technol., v. 100, p. 5818-5826, 2009.

FORTES, I. C. P.; BAUGH, P. J. Pyrolysis-GC/MS studies of vegetable oils from macauba fruit. $J$. Anal. Appl. Pyrolysis. v. 72, p. 103-111, 2004.

HIANE, P. A.; RAMOS FILHO, M. M.; RAMOS M. I. L.; MACEDO, M. L. R. Bocaiúva, Acrocomia aculeata (Jacq.) Lodd., Pulp and Kernel Oils: Characterization and Fatty Acid Composition. Braz. J. Food Technol., v. 8, p. 256-259, 2005.

GONZALES, L. S. Produção contínua de biodiesel por transesterificação de óleo do fruto de macaúba (acrocomia aculeata) e óleo de fritura em metanol e em etanol supercrítico. Tese de Doutorado. Universidade Federal de Santa Catarina - UFSC, Florianópolis, SC, 2012.

ISSARIYAKUL T.; KULKARNI M.G.; MEHER L.C.; DALAI A.K.; BAKHSHI N.N. Biodiesel production from mixtures of canola oil and used cooking oil, Chem. Eng. J., v. 140, p. 77, 2008.

MARULANDA V.F.; ANISTESCU G.; TAVLARIDES L.L. Biodiesel fuels through a continuous flow process of chicken fat supercritical transesterification, Energy \& Fuels, v. 24, p. 253, 2010.

NOGUEIRA, B. M.; CARRETONI, C.; CRUZ, R.; FREITAS, S.; MELO, P. A.; FÉLIX-COSTA, R.; PINTO, J. C.; NELE, M. Microwave activation of enzymatic catalysts for biodiesel production. J. Mol. Catal. B: Enzym, v. 67, p. 117-121, 2010.

RUIZ-MÉNDEZ, M. V.; MARMESAT, S.; LIOTTA, A.; DOBARGANES, M. C. Analysis of used frying fats for the production of biodiesel. Grasas y aceites. v. 59. n.1, p. 45-50, 2008.

STANDARD UNE-EN 14103. Fat and oil oil derivatives: Fatty Acid Methyl esters (FAME) Determination of ester and linolenic acid methyl ester contents. Asociación Española de Normalización y Certificación, Madrid, 2003.

THANH L.T.; OKITSU K.; SADANAGA Y.; TAKENAKA N.; MAEDA Y.; BANDOW H. A twostep continuous ultrasound assisted production of biodiesel fuel from waste cooking oils: A practical and economical approach to produce high quality biodiesel fuel. Bioresource Technol., v. 101, p. 5394, 2010.

YU, D.; TIAN, L.; WU, H.; WANG, S.; WANG, Y.; MA, D.; FANG, X. Ultrasonic irradiation with vibration for biodiesel production from soybean oil by Novozym 435. Process. Biochem., v. 45, p. 519-525, 2010. 